\title{
Computer Simulation Based on 3D Printer
}

\author{
Liang Dong ${ }^{1, a}$ \\ ${ }^{1}$ Beijing Institute of Technology, Beijing, 100081, China \\ aDong_630@msn.com
}

Keywords: 3D printing; computer simulation; temperature field; thermodynamics

\begin{abstract}
This paper is based on 3D printing technology, as for study and design on those printing tests; it develops a set of 3D printing equipment to make design on head, modelling chamber and temperature system. It simulates temperature field in 3D printing process and provides instruction for 3D printing test to analyse heat stress coupling field. The study results indicate that the higher temperature in head and modelling chamber, the quicker speed pf printing, the higher overall temperature on printing, the smoother of temperature distribution. Modelling chamber temperature and printing speed have stronger effect on model temperature field, while head temperature has little effect.
\end{abstract}

\section{Introduction}

After human beings evolving up to now, one new civilization symbol is to use machine to produce machine [1, 2]. From the narrow sense, 3D printer endowed with unlimited imaginations to use machine to produce machine. It enables 3D entity in the objective world to realize reconstruction of high-precision (3D scanning), free editing (3D design), real HD display (3D display), even returns to objective world (3D printing) [3, 4]. In the future, 3D printer will develop toward intelligent, HD, multi-material and compound printing direction etc. For example, intelligent identification and feed back function will be the problems urgently need to be solved for quick modelling system, through process parameter accumulation; it makes 3D printing equipment automatically make judgment by itself, intelligently adjust and optimize process parameter, making complicated quick modelling system become simple and have practicability and popularization. Under data cloud support of intelligent printing, 3D printing robot becomes to be cleverer, enabling 3D printing technology to easy for popularization and promotion. Because of technology popularization, the application cost should be reduced after large-scaled application, and popularization and application of 3D printing technology are in progress, many printing material are very expensive, it needs longer time to go into family users, people need to urgently to make study on 3D printing technology for problems such as invasion on intellectual property right and challenges on moral and human relations.

This paper is based on different 3D printing to realize the common characteristics under technology difference, seeking to fund one universal simulation method for 3D printing process, making this kind of technology can be easily transferred and promote to other kinds of technologies in 3D printing, so it can further develop performance analysis and prediction of 3D printer performance analysis and prediction with uniform panel and function, making intelligent software of printing software to be possible.

\section{3D Study and development of printing test}

\subsection{Overall design of system}

This system includes one 3D printing platform moves towards X, Y direction, the platform surface pastes PEEK film of $0.2 \mathrm{~mm}$. The 3D printing modelling chamber with heating enclosing is installed on the 3D printing platform, the modelling chamber uses stainless steel of $0.5 \mathrm{~mm}$ as shell, inside it installs nicochrome resistance wire as heating element and encloses by insulated and high-temperature resistance micarex. Head and PEEK wire feed system move upwards and 
downwards along $\mathrm{Z}$ axle. The temperature control system of this unit includes head temperature control system, modelling chamber temperature control system and printing platform temperature control system. The basic parameter is as follows: thickness of printing layer: $200 \mu \mathrm{m}-400 \mu \mathrm{m}$, head diameter: $0.4 \mathrm{~mm}$, construction speed can reach $15 \mathrm{~cm}$ for 3 hours, the dimension of modelling space: $120 \mathrm{~mm} \times 120 \mathrm{~mm} \times 120 \mathrm{~mm}$, printing material is wire material with diameter of $1.65 \mathrm{~mm}$.

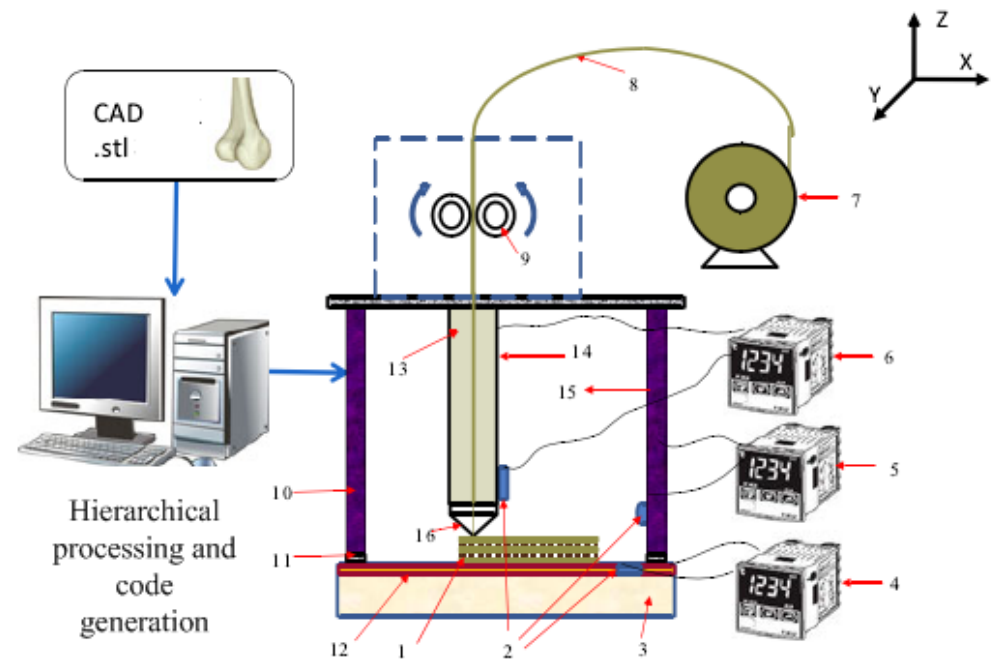

Diagram 1 Work principle diagram of 3D printing system

\subsection{Design on system head and base board of modelling chamber for 3D printing system}

The designed head of this test adopts separate external hearing way, it installs built-in single heating rod in the external part of plastify cavity to realize melting of wire material. Because melting point of wire material is higher and viscosity is larger, in order to fully make it plastify in the printing process, it lengthens length of head material feed tube, designs close-cycle heating system of double hearing, and adopts heating way by sections, pre-heating of feed inlet, full heating in material tube so that it can guarantees complete plastify of material. It designs temperature sensor near head to test the real-time temperature of head and it can realize thermostatically control by temperature controller. The diameter of material feed tube is $1.65 \mathrm{~mm}$, the internal diameter is $2 \mathrm{~mm}$, this special design can reduce air flow in the wire feed process, meanwhile, it guarantees full melting material storage and complete plastify in the feed, reduce bubble situation in wire out, the head diameter is $400 \mu \mathrm{mm}$ rectify small step surface and guarantees quality of printing parts.

The base board of printing has $\mathrm{X}$ and $\mathrm{Y}$ free degree, modelling chamber can move with base board, this layout can make printer accessories avoid high temperature melting outside modelling chamber and influence its work performance, the modelling chamber adopts stainless steel heating box as external wall, using insulated and high-temperature resistance micarex as core in the middle part, it winds nicochrome resistance wire as heating element of all kinds of standard and specification, which forms base board carrying movement.

\subsection{Establishment of temperature control system}

Process of realizing 3D printing needs to control temperature adjustment and constant of 3 ways, including head temperature and modelling chamber temperature and heating platform temperature, its temperature control system can adopt the same plan to realize. In order to reduce development cost and time, this system directly adopts temperature controller, silicon control, heating rod, stainless steel heating ring and thermocouple of human-machine interface with high precision as hardware parts of system (diagram 2), it composes independent close-cycle temperature control system to realize precise and correct control on printer head, modelling chamber and heating platform temperature (diagram 3). 


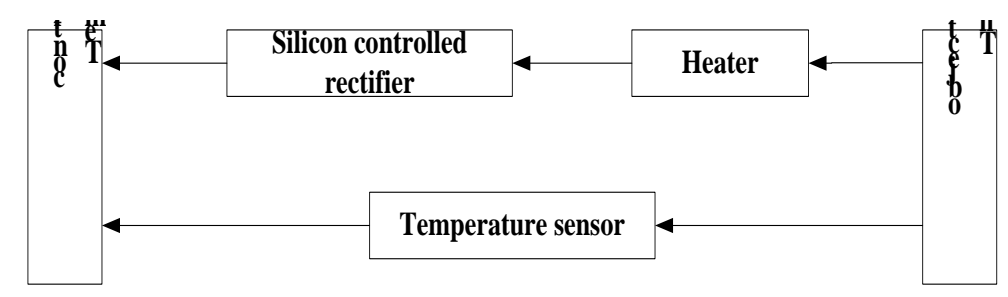

Diagram 2 Hardware diagram of temperature control system

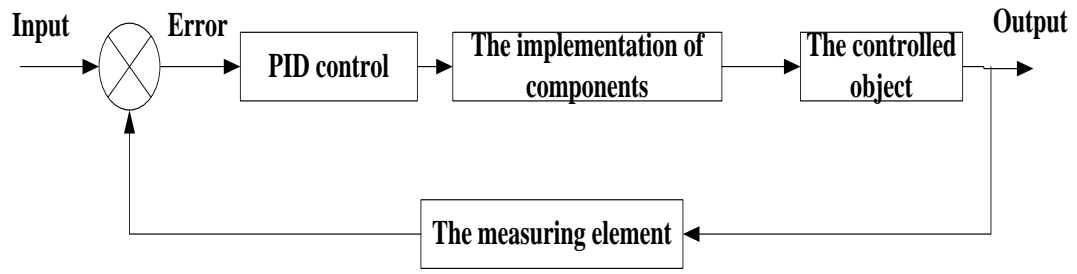

Diagram 3 Indication chart of close-cycle control

The temperature controller in this equipment adopts OMRON industrial temperature controller E5CC-QX2ASM-802, it has white-light human-machine interface with height of $15.2 \mathrm{~mm}$, and the display precision is $\pm 1 \mathrm{C}$, the sampling period $50 \mathrm{~ms}$. The volume is very small and installation is simple. The silicon control switch adopts solid state relay of OMRON G3NA series, it adopts simulation of photo coupler, the output applicable load is $10 \mathrm{~A}$, DC5V-200V, the input rated voltage is DC5V-24V, equips with radiator of Y92B-N50, temperature sensor adopts nichrome-nisiloy thermocouple of K type.

\section{Temperature Field Simulation Based on 3D Printing Process}

\subsection{D Temperature field model and process of printing}

3D printing material adopts special engineering plastic PEEK of heat-resistant and heat plastic, in the printing process, PEEK density will change with crystallization degree, its material property indicates non-linear characteristic with temperature change. It uses $12 \times 12 \times 1.2 \mathrm{~mm}$ cube of filmy board as object to make finite element analysis, it adopts 3D entity heat unit SOLID70 used for temperature field calculation in ANSYS to divide grid. According to head wire width in the real printing process to confirm that there are 1600 cuboids of $0.6 \times 0.6 \times 0.3 \mathrm{~mm}$ to make grid division, it will activate one unit after one load step time, it will complete the whole 3D printing process after activating 1600 steps according to given scanning locus.

Before 3D printing, there should be enough preheating for long time to reach given temperature, suppose its internal part has the same temperature through pre-heating, the initial condition is as follows:

$$
\left.T(x, y, z)\right|_{t=0}=T_{0}
$$

The radiation way on sample surface is comprehensive effect of heat convection and heat radiation, the calculated heat exchange parameter $\mathrm{h}$ is as follows:

$$
h=\sigma \varepsilon\left(T_{p}^{2}+T_{c}^{2}\right)\left(T_{p}+T_{c}\right)+h_{c}
$$

In the formula, hc is the heat exchange parameter of natural convection, $(\mathrm{W} / \mathrm{m} 2 \mathrm{C}) ; \sigma-$ Stefan-Boltzman constant, its value is $5.67 \times 10-8 \mathrm{~W} / \mathrm{m} 2 \cdot \mathrm{K} 4 ; \varepsilon$-black degree of sample material; $\mathrm{Tp}$-temperature of sample part, (C) ; Tc-environment temperature, (C)。

This paper adopts unit death technology in finite element and applies ADDL simulation material moves according to scanning locus under the given printing conditions, it will activate corresponding 
unit and provide material and heat source at different times. The flow chart of 3D printing temperature field is indicated by diagram 4.

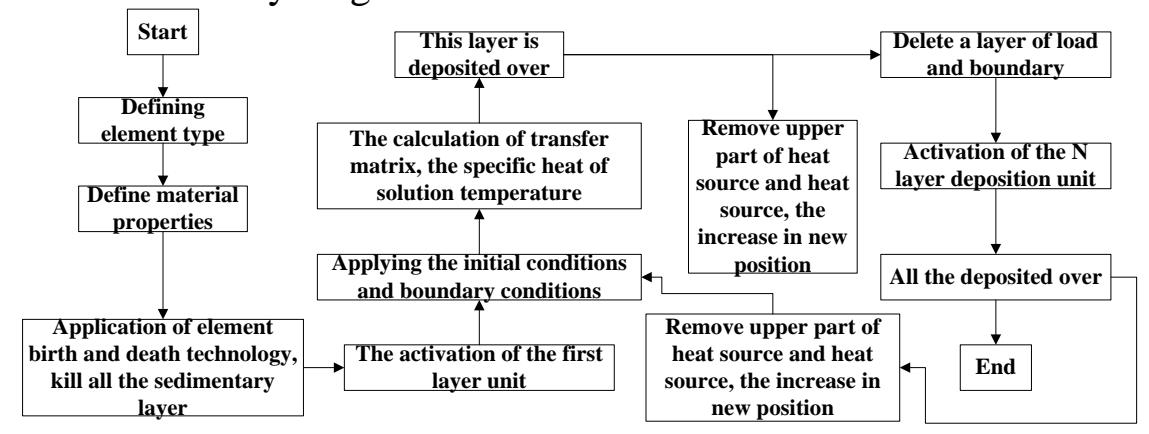

Diagram 4 Finite element flow chart of temperature field on 3D printing is completed

\subsection{Simulation result of temperature field during printing}

During 3D printing, sample node will change with time change; overall distribution condition has certain relations to head temperature, modelling chamber temperature, printing speed. In order to intuitively analyse influence of different printing conditions on distribution rule of sample part temperature, it will predict sample part deformation, defect and choose proper printing conditions to guide test, respectively programmes command stream on each printing conditions and make finite element analysis on temperature field, it makes analysis on temperature field distribution, temperature gradient change trend and temperature change ratio result of node. Table 1 indicates the analysis condition.

Table 1 Process parameter of 3D printing under different printing conditions

\begin{tabular}{|c|c|c|c|c|c|c|c|c|c|c|}
\hline \multicolumn{3}{|c|}{$\begin{array}{c}\text { Head } \\
\text { Temperature } \\
\text { (C) }\end{array}$} & \multicolumn{4}{|c|}{$\begin{array}{l}\text { Modelling chamber } \\
\text { temperature (C) }\end{array}$} & \multicolumn{4}{|c|}{ Printing speed $(\mathrm{mm} / \mathrm{s})$} \\
\hline 340 & 350 & 360 & 90 & 110 & 130 & 220 & 20 & 30 & 40 & 100 \\
\hline
\end{tabular}

After simulation answer analysis is completed, we can check answer result of different time point by postprocessor POST1 of ANSYS generalized time, in addition, we can clearly see distribution condition of temperature field with time change by cloud chart or list table. In this analysis, the printing condition is as follows: head temperature $\mathrm{T} 1=350 \mathrm{C}$, modelling chamber temperature $\mathrm{T} 2=130 \mathrm{C}$, printing speed $\mathrm{V}=30 \mathrm{~mm} / \mathrm{s}$, this printing speed is the usual speed of $3 \mathrm{D}$ printing equipment, the layer thickness $\mathrm{H}=300 \mu \mathrm{m}$, divides analysis model into 4 layers. The scanning route adopts cross grid scanning method. The printing time totals 32s, the unit activation time is $0.02 \mathrm{~s}$, and the time of completing one scanning line is $0.4 \mathrm{~s}$. Model goes into cooling state after $32 \mathrm{~s}$, it cools down to indoor temperature after $63 \mathrm{~s}$. Diagram 5-8 are respectively corresponding time of each layer during printing $6.4 \mathrm{~s}, 11.2 \mathrm{~s}, 21 \mathrm{~s}$ and $30 \mathrm{~s}$ and distribution condition of temperature field after printing is ended during cooling process in 33.6s, 40s, 62s. 

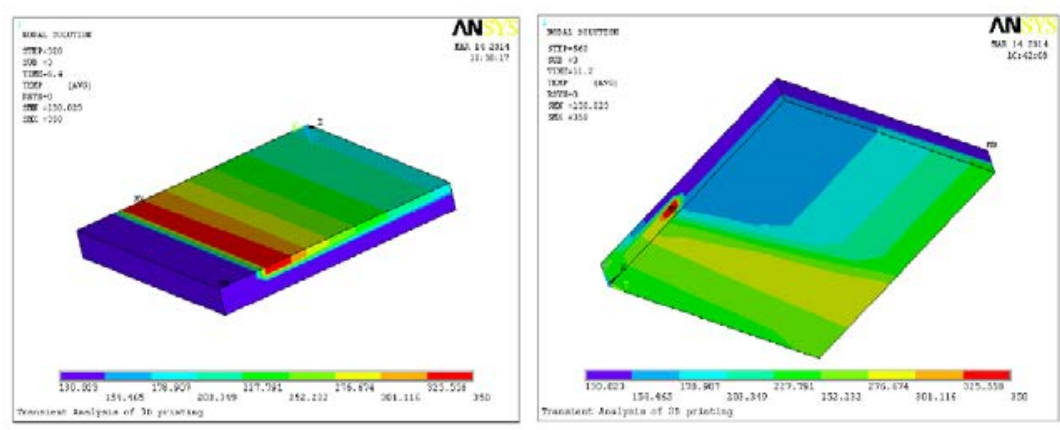

Diagram 5 Temperature field of printing the 1st layer Diagram 6 Temperature field of printing the 2nd layer
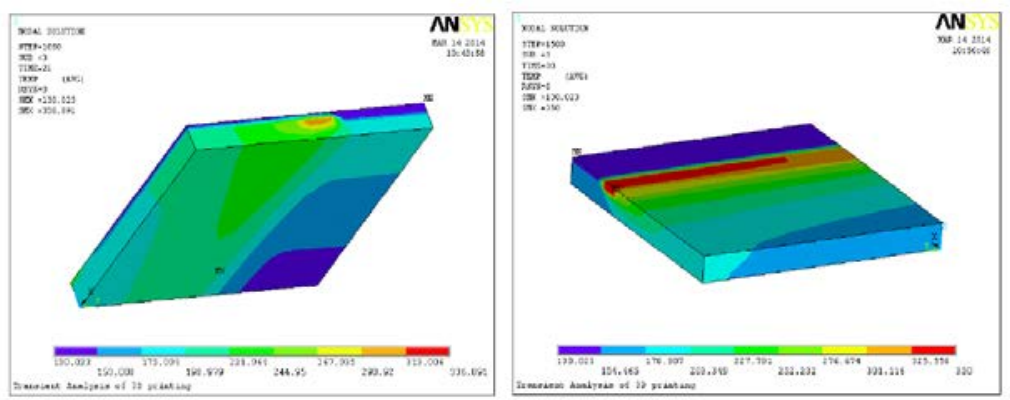

Diagram 7 Temperature field when printing the 3rd layer Diagram 8 Temperature field of printing the 4th layer

After printing is completed, the temperature field of model in different times is indicated by diagram 9 and diagram 10.

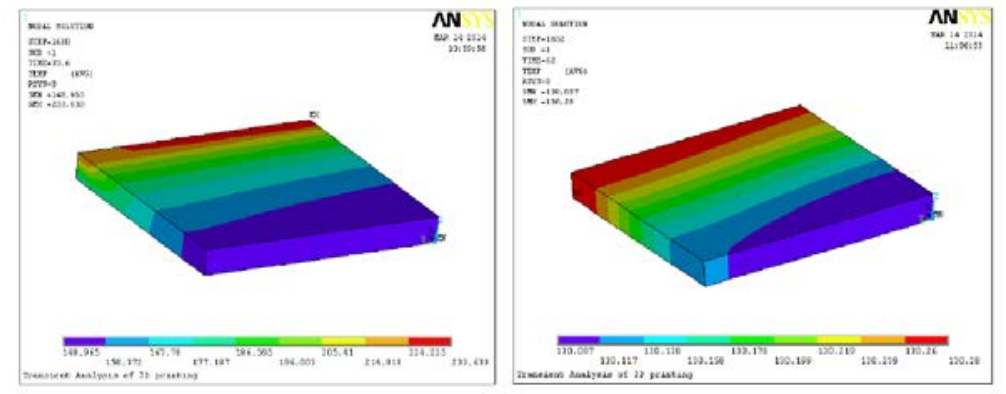

Diagram 9 Temperature field cooled to 33.6s Diagram 10 Temperature field cooled to 62s

When printing the first layer, because the upper material has not been generated, it is the killed state, its temperature is the same with modelling chamber, temperature instantly increase on position of head movement, the already formed part exchanges heat by heat conduction and surface convection to exchange heat, so temperature gradually decreases. With printing is in progress, heat influence areas are gradually enlarging, the isotherm at current printing layer is dense and spreads by band shape, the isotherm at lower is sparse, shape is irregular, at the same time, the overall temperature distribution of model is uneven and change is relatively bigger. After printing is completed, model temperature quickly decreases, until all point tends to form indoor temperature.

\subsection{Influence analysis of printing condition on temperature field distribution}

From diagram 11, we can see that after printing is completed, model temperature of different head temperature has the same distribution rule in model isotherm under head temperature, with increase in head temperature, the final printing temperature range enlarges, but the lowest temperature relatively increases, the overall change is less than $1 \mathrm{C}$. Changing head temperature has little effect on 
overall temperature field distribution of model after printing is completed.

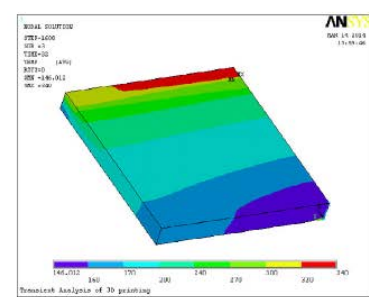

(a) $\mathrm{T} 1=340 \mathrm{C}$

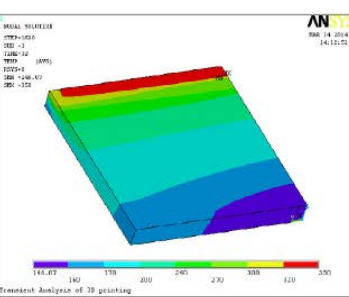

(b) $\mathrm{T} 1=350 \mathrm{C}$

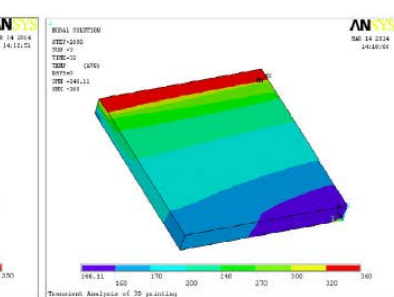

(c) $\mathrm{T} 1=360 \mathrm{C}$

Diagram 11 Temperature field distribution when model printing is completed under diferent head temperatuer

This paper chooses the usual printing speed of $40 \mathrm{~mm} / \mathrm{s}$ and design target of $100 \mathrm{~mm} / \mathrm{s}$ as simulation condition to make finite element analysis, the activation time of corresponding unit is respectively 0.03s, 0.02s, 0.015s and 0.006s. According to overall temperature distribution condition of model when printing is completed, it will predict suitable printing speed range and feasibility of high-speed printing. Diagram 12 is temperature field distribution condition on printing is completed at different printing speed. With increase in printing speed, overall temperature of model will increase on printing is completed, from temperature field distribution, the surface temperature range of model increases, but overall temperature difference reduces, temperature distribution is relatively even.

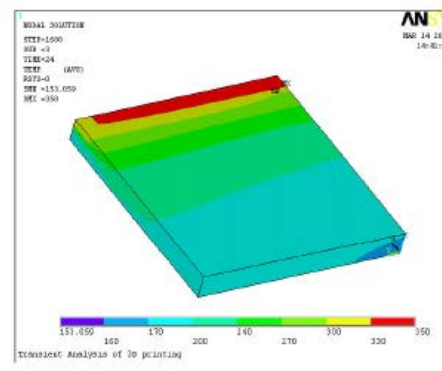

(c) $\mathrm{V}=40 \mathrm{~mm} / \mathrm{s}$

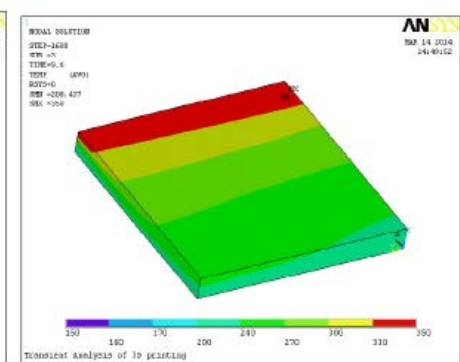

(d) $V=100 \mathrm{~mm} / \mathrm{s}$

Diagram 12 Temperature field when model printing is completed at different printing speed

\subsection{Influence analysis on temperature gradient of characteristic point by printing speed}

As it is indicated by diagram 13, we can see that different printing speed has great influence on temperature gradient, with increase in printing speed, temperature gradient value obviously decreases, when printing speed $\mathrm{V}=100 \mathrm{~mm} / \mathrm{s}$, on precondition of without affecting process, the printing condition should possibly choose head temperature, high modelling chamber temperature and increase printing speed in order to reduce temperature gradient of characteristic point, sample deformation and guarantee modelling quality. 


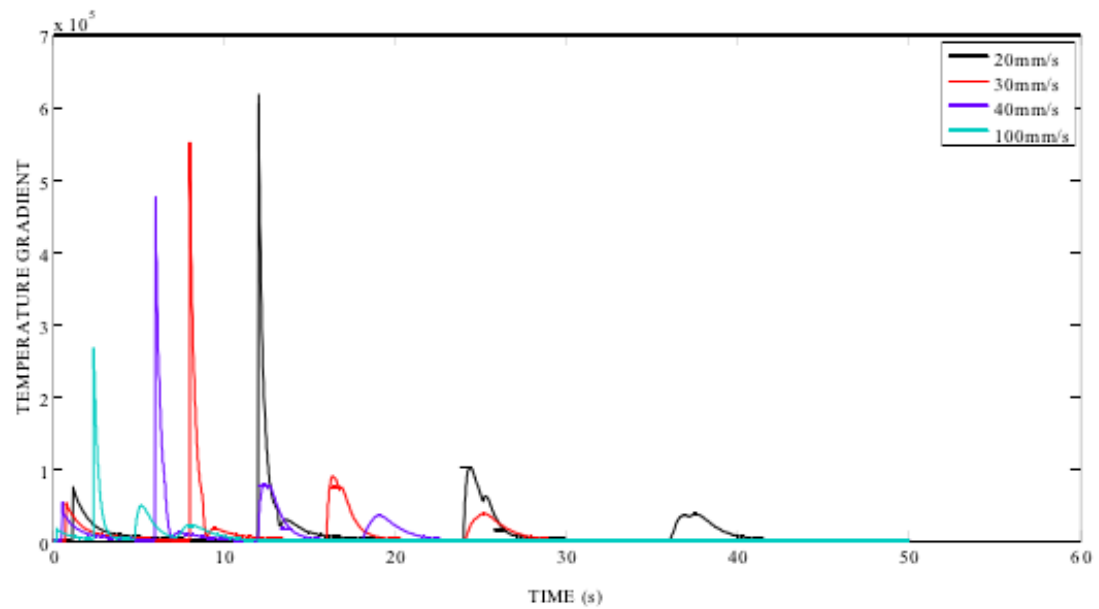

Diagram 13 Chnage curve of characteristic point temperature gradient under different printing speed

\section{Conclusions}

This paper makes profound analysis on heat act of FDM 3D printing process, discloses influence rule of printing conditions on sample part, analyses distribution condition of temperature, change rule of node temperature with time, temperature gradient characteristic, node cooling time and cooling ratio in the printing process, it also analyses influence rule of changing head temperature, modelling chamber, printing speed on temperature field of sample parts, indicates that the higher of head temperature and modelling chamber, the quicker of printing speed., the higher of overall model after printing, the smoother of temperature distribution, modelling chamber temperature and printing speed will have stronger influence on model temperature field, head temperature has little effect.

\section{References}

[1] Wang Xueying. Analysis on 3D Printing Technology as well as Industry Development and Prospect. China Hi-tech Enterprise, 2012,233(26):3-5.

[2] Liu Hongguang, Yang Qian, Liu Guifeng etc. Patent and Situation Analysison 3D Princting Quick Modeling both in China and Abroad. Situation Magazine, 2013, 32(6): 40-46.

[3] Wang Cancai. Analysis on Development State of 3D Printing [J]. Creen Print, 2012(9): 37-41.

[4] Zhao HH,Zhang GJ,Yin ZQ,Wu L (2011) A 3D dynamic analysis of thermal behavior during single-pass multi-layer weld-based rapid prototyping. J Mater Process Technol 211(3):488^95

[5] Lutz M. Learning python [M]. ', O'Reilly Media,Inc.", 2013.

[6] Wang Zhonghong, Li Yangfan, Zhang Manyin. State and Development Idea of Chinese 3D Printing. Economic Aspect 2013,1: 90-93.

[7] Liu Weijun. Quick Modeling Technology and Application [M].Beijing: Machinery Industry Press, 2005.

[8] Hu Di, Lipson, Melba)Kuman.3D Printing: from Imagination to Reality [M]. CITIC Publishing House, 2013.

[9] Ai Senzhe. 3D Printing Makes Longer [M]. China Commercial Circle， 2013， (08):110-111.

[10] Zhang Yangya, Li Daguang. 3D Printing Technology will Cause Reforming Arm Equipment [M].Arm Equipment, 2013, (07): 54-55. 